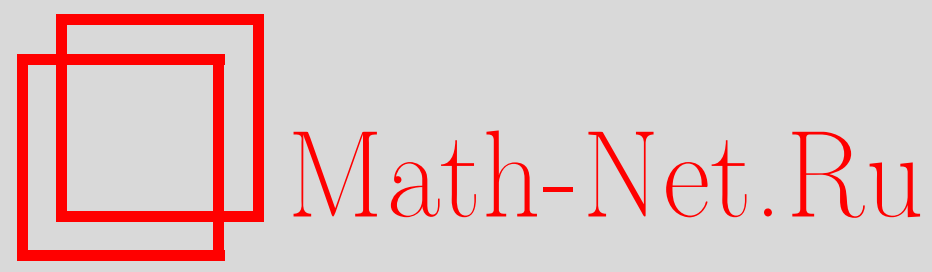

А. В. Шаповалов, Характеристики случайных систем линейных уравнений над конечным полем, Дискрет. матем., 2008, том 20, выпуск 4, 136-146

DOI: https://doi.org/10.4213/dm1033

Использование Общероссийского математического портала Math-Net.Ru подразумевает, что вы прочитали и согласны с пользовательским соглашением http://www . mathnet.ru/rus/agreement

Параметры загрузки:

IP : 34.239 .49 .27

26 апреля 2023 г., $18: 23: 31$

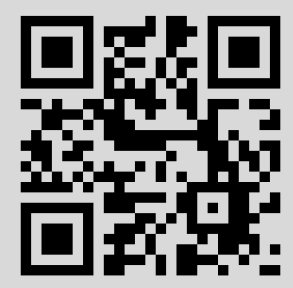




\title{
Характеристики случайных систем линейных уравнений над конечным полем
}

\author{
() 2008 г. А. В. Шаповалов
}

\begin{abstract}
Рассматриваются случайная и заведомо совместная случайная системы линейных уравнений над конечным полем из $q$ элементов относительно $n$ неизвестных. Случайные системы состоят из $M=M(n)$ уравнений, каждое из которых может зависеть от $2,3, \ldots, m$ переменных, выбираемых случайно, равновероятно и бесповторно. Получены предельные распределения и оценки моментов чисел решений случайных систем уравнений при $n \rightarrow \infty$, когда соотношение параметров $n$ и $M$, числа вершин и числа гиперребер, определяет докритическую область эволюции случайных гиперграфов, сопоставляемых случайным системам уравнений. Вид и параметры предельных распределений определяются характеристиками предельных распределений чисел циклов специального вида в случайных гиперграфах.
\end{abstract}

\section{1. Формулировка результатов}

Для каждого $i=2, \ldots, m$ через $L_{i}=\left\{f_{i, 1}, f_{i, 2}, \ldots, f_{i,\left|L_{i}\right|}\right\}$ обозначим некоторым образом упорядоченное множество всех линейных функций над конечным полем из $q>2$ элементов $G F(q)$, существенно зависящих ровно от $i$ переменных, причем $f_{2,1}\left(y_{1}, y_{2}\right)=y_{1}-y_{2}$, $f_{2,2}\left(y_{1}, y_{2}\right)=y_{1}+y_{2}$. Определим неотрицательные константы $c_{2}, \ldots, c_{m}, c_{2,1}, c_{2,2}$, удовлетворяющие условиям $c_{2}+\ldots+c_{m}=1, c_{2}=c_{2,1}+c_{2,2}>0$, пусть $\Delta=c_{2,2}-c_{2,1}$. Формула $\xi_{n} \stackrel{d}{\longrightarrow} \xi$ означает сходимость по распределению при $n \rightarrow \infty$ последовательности случайных величин $\left\{\xi_{n}\right\}$ к случайной величине $\xi, P\left(\lambda_{1}, \ldots, \lambda_{t}\right)-$ многомерное распределение Пуассона с параметрами $\lambda_{1}, \ldots, \lambda_{t},(z)_{k}=z(z-1) \ldots(z-k+1)$. Все асимптотические формулы приводятся при $n \rightarrow \infty$.

В случайном уравнении над $G F(q)$

$$
f_{i, j}\left(x_{s_{1}}, \ldots, x_{s_{i}}\right)=a
$$

относительно $n$ неизвестных $x_{1}, \ldots, x_{n}$ выбор упорядоченного множества индексов неизвестных $\left\{s_{1}, \ldots, s_{i}\right\}$ осуществляется случайно и равновероятно по схеме с возвращением из всех $(n)_{i}$ возможных упорядоченных наборов индексов по $i$ штук, $i=2, \ldots, m$. При $i=2$ выбор значений 1 и 2 индекса $j$ (то есть функций $y_{1}-y_{2}$ и $y_{1}+y_{2}$ ) осуществляется с вероятностями $c_{2,1} / c_{2}$ и $c_{2,2} / c_{2}$. При $i=2, j=2$ упорядоченные выборки $\left\{x_{s_{1}}, x_{s_{2}}\right\}$ и $\left\{x_{s_{2}}, x_{s_{1}}\right\}$ не различаются и объединяются в один исход. При каждом фиксированном $i, i=3, \ldots, m$, выбор значения индекса $j$ (то есть функции из $L_{i}$ для левой части уравнений) и значения правой части уравнений из $G F(q)$ осуществляется по каким-то вероятностным законам. 
Случайное уравнение (1) удовлетворяет условию $\mathbf{B}$, если его правая часть принимает значения $0,1, \ldots, q-1$ с равными вероятностями.

В случайном заведомо совместном уравнении над $G F(q)$

$$
f_{i, j}\left(x_{s_{1}}, \ldots, x_{s_{i}}\right)=f_{i, j}\left(b_{s_{1}}, \ldots, b_{s_{i}}\right)
$$

выбор левой части определяется так же, как и в случайном уравнении (1), $i=2, \ldots, m$, а правая часть каждой реализации случайного уравнения равна значению функции в ее левой части, получаемому при подстановке вместо $x_{1}, \ldots, x_{n}$ вектора $\mathbf{b}=\left(b_{1}, \ldots, b_{n}\right) \mathrm{c}$ компонентами из $G F(q)$, называемого истинным решением.

Случайные системы линейных уравнений $S=S_{n}$ и $S^{(c)}=S_{n}^{(c)}$ относительно $n$ неизвестных $x_{1}, \ldots, x_{n}$ состоят из $M=M(n)$ случайных уравнений вида (1) и (2) соответственно, причем $i=2$ для первых $M_{2}$ уравнений, $i=3$ для следующих $M_{3}$ уравнений, и так далее, и $i=m$ для последних $M_{m}$ уравнений. В случайной системе уравнений $S$ первые $M_{2}$ случайных уравнения удовлетворяют условию В. Числа случайных уравнений вида (1) и (2) в случайных системах $S$ и $S^{(c)}$ удовлетворяют соотношениям $M_{i}=0$ при $c_{i}=0$ и $M_{i} \sim c_{i} M$ при $c_{i}>0, i=2, \ldots, m$.

Обозначим через $\zeta_{n}$ и $\zeta_{n}^{(c)}$ числа решений случайных систем уравнений $S$ и $S^{(c)}$, деленные на $q^{n-M}$, а через $\zeta$ и $\zeta^{(c)}-$ случайные величины, распределения которых описываются следующими образом:

$$
\begin{aligned}
& \mathbf{P}(\zeta=0)=1-e^{-\mu^{(0)}}=1-\left(\sqrt[4]{\left(1-2 c c_{2}\right)(1+2 c \Delta)} e^{c c_{2,1}}\right)^{(q-1) / q}, \\
& \mathbf{P}\left(\zeta=q^{k}\right)=e^{-\mu^{(0)}-\mu^{(q)}}\left(\mu^{(q)}\right)^{k} / k !, \quad k=0,1, \ldots, \\
& \mathbf{P}\left(\zeta^{(c)}=q^{k}\right)=e^{-\lambda} \lambda^{k} / k !, \quad k=0,1, \ldots,
\end{aligned}
$$

где

$$
\lambda=-\frac{1}{4} \log \left(\left(1-2 c c_{2}\right)(1+2 c \Delta)\right)-c c_{2,1}, \quad \mu^{(q)}=\frac{\lambda}{q}, \quad \mu^{(0)}=\frac{\lambda(q-1)}{q} .
$$

Легко видеть, что для $r=1,2, \ldots$

$$
\begin{gathered}
\mathbf{E} \zeta^{r}=e^{-\mu^{(0)}+\mu^{(q)}\left(q^{r}-1\right)}=\left(\sqrt[4]{\left(1-2 c c_{2}\right)(1+2 c \Delta)} e^{c c_{2,1}}\right)^{-\left(q^{r-1}-1\right)}, \\
\mathbf{E}\left(\zeta^{(c)}\right)^{r}=e^{\lambda\left(q^{r}-1\right)}=\left(\sqrt[4]{\left(1-2 c c_{2}\right)(1+2 c \Delta)} e^{c c_{2,1}}\right)^{-\left(q^{r}-1\right)} .
\end{gathered}
$$

Следующие две теоремы являются основными результатами работы.

Теорема 1. Если $n \rightarrow \infty u$

$$
M \sim c n, \quad 0<c<c^{*}=\left(c_{2}(2)_{2}+c_{3}(3)_{2}+\ldots+c_{m}(m)_{2}\right)^{-1},
$$

то $\zeta_{n} \stackrel{d}{\longrightarrow} \zeta, \underline{\lim }_{n \rightarrow \infty} \mathbf{E} \zeta_{n}^{r} \geqslant \mathbf{E} \zeta^{r}$, причем $\mathbf{E} \zeta_{n}^{r} \rightarrow \mathbf{E} \zeta^{r}, r=1,2, \ldots$, при выполнении условия В для всех уравнений случайной системь $S$.

Теорема 2. Если $n \rightarrow \infty$ и выполнено условие (3), то $\mathbf{E}\left(\zeta_{n}^{(c)}\right)^{r} \rightarrow \mathbf{E}\left(\zeta^{(c)}\right)^{r}, r=1,2, \ldots, u$ $\zeta_{n}^{(c)} \stackrel{d}{\longrightarrow} \zeta^{(c)}$.

В данной работе, как и в [4, 7, 9-11], характеристики случайных систем линейных уравнений получаются на основе изучения структуры компонент связности и распределений чисел циклов и гиперциклов случайных графов и гиперграфов в докритической 
области эволюции. Случайной системе уравнений сопоставляется случайный гиперграф, каждой ее реализации - реализация случайного гиперграфа (см. [2, 4, 7, 9-12]). При этом переменной $x_{k}$ соответствует вершина $v_{k}, k=1, \ldots, n$, а каждое уравнение системы однозначно определяет соответствующее ему ребро сопоставляемого системе гиперграфа: ребро состоит из вершин, соответствующих существенным переменным функции в уравнении. Порядок на множестве вершин ребра определяется порядком на множестве неизвестных соответствующего уравнения. Ребро с меткой $(i, j, a)$ соответствует уравнению с функцией $f_{i, j}$ в левой части и элементом $a$ в правой части. Установленное соответствие между системами уравнений и гиперграфами взаимно однозначно. Случайным системам уравнений $S$ и $S^{(c)}$ сопоставляются случайные гиперграфы $M G(S)$ и $M G\left(S^{(c)}\right)$, реализациями которых являются гиперграфы с множеством вершин $\left\{v_{1}, \ldots, v_{n}\right\}$ и $M$ ребрами. Вершины в ребрах упорядочены (при $i=2$ могут быть и неупорядочены), ребра имеют метки вида $(i, j, a)$. Вероятности реализаций случайных гиперграфов равны вероятностям соответствующих им реализаций случайных систем уравнений.

Справедливы следующие эквивалентные определения рассматриваемых случайных систем уравнений и соответствующих им случайных гиперграфов. На первом этапе для каждого $i=2, \ldots, m$ последовательно и независимо друг от друга для $M_{i}$ уравнений (ребер) выбираются $i$ неупорядоченных переменных (вершин) с одинаковыми вероятностями из всех $\left(\begin{array}{c}n \\ i\end{array}\right)$ возможных вариантов. На втором этапе с вероятностями $c_{2,1} / c_{2}$ и $c_{2,2} / c_{2}$ для левой части первых $M_{2}$ уравнений выбираются функции $y_{1}-y_{2}$ и $y_{1}+y_{2}$, их правые части $а$ выбираются равновероятно из $G F(q)$ (ребрам приписываются метки $(2,1, a)$ и $(2,2, a))$, в уравнениях с функцией $y_{1}-y_{2}$ (в ребрах с меткой вида $\left.(2,1, a)\right)$ случайно и равновероятно выбирается порядок переменных (вершин). Затем при каждом $i, i=3, \ldots, m$, для следующих $M_{i}$ уравнений (ребер) выбираются номер $j$ функции из $L_{i}$ и значения правых частей уравнений из $G F(q)$ (приписываются метки $(i, j, a)$ ) в соответствии с вероятностными мерами случайных уравнений (1) и (2), а множество выбранных на первом этапе переменных (вершин) упорядочивается случайно и равновероятно.

Такие определения случайных элементов позволяют сделать вывод, что для первоначального изучения их геометрической структуры без детализации, учитывающей конкретный вид функций и правые части уравнений, достаточно знания величин $c_{2}, \ldots, c_{m}$. Если в таких определениях случайных гиперграфов $M G(S)$ и $M G\left(S^{(c)}\right)$ остановиться после первого этапа, то получится случайный гиперграф $M G_{n, \bar{M}}$, свойства которого изучались в работах [9, 11-14], а при $c_{2}=c_{2,2}=1-$ в [4, 5]. Следовательно, эти результаты справедливы для $M G(S)$ и $M G\left(S^{(c)}\right)$. Так, из теоремы 1 в [14] следует, что при условии (3) случайные гиперграфы со стремящейся к единице вероятностью не имеют компонент с двумя и более циклами.

Гиперграф $F$ называется подграфом гиперграфа $H$, если множества вершин и ребер $F$ являются подмножествами множеств вершин и ребер $H$. Изоморфный гиперграфу $F$ подграф гиперграфа $H$ называется $F$-графом. Под 2-циклами в данной работе понимаются гиперграфы, которые в теории графов называются циклами.

Пусть $H_{1}, \ldots, H_{t}$ и $H_{1}^{(c)}, \ldots, H_{t}^{(c)}-$ фиксированные неизоморфные 2-циклы с метками ребер в гиперграфах $M G(S)$ и $M G\left(S^{(c)}\right)$, гиперграфы $H_{k}$ и $H_{k}^{(c)}$ имеют по $l^{(k)}$ вершин, $l_{2,2}^{(k)}$ ребер с неупорядоченными вершинами, $l_{2,1}^{(k)}=l^{(k)}-l_{2,2}^{(k)}$ ребер с упорядоченными вершинами, $0 \leqslant l_{2,2}^{(k)} \leqslant l^{(k)}, l^{(k)} \geqslant 2$, порядки групп автоморфизмов $a_{k}$ и $a_{k}^{(c)}, \xi_{k}=\xi_{k, n}$ и $\xi_{k}^{(c)}=\xi_{k, n}^{(c)}$ - числа $H_{k}$-графов в $M G(S)$ и $H_{k}^{(c)}$-графов в $M G\left(S^{(c)}\right)$, $k=1, \ldots, t, t \geqslant 1$. 
Теорема 3. Если $n \rightarrow \infty, M \sim c n$, где $c>0-$ константа, то

$$
\begin{array}{cr}
\left(\xi_{1}, \ldots, \xi_{t}\right) \stackrel{d}{\longrightarrow} P\left(\gamma_{1}, \ldots, \gamma_{t}\right), & \left(\sum_{i=1}^{t_{1}} \xi_{i}, \sum_{k=t_{1}+1}^{t} \xi_{k}\right) \stackrel{d}{\longrightarrow} P\left(\sum_{i=1}^{t_{1}} \gamma_{i}, \sum_{k=t_{1}+1}^{t} \gamma_{k}\right), \\
\left(\xi_{1}^{(c)}, \ldots, \xi_{t}^{(c)}\right) \stackrel{d}{\longrightarrow} P\left(\gamma_{1}^{(c)}, \ldots, \gamma_{t}^{(c)}\right), & \sum_{i=1}^{t} \xi_{i}^{(c)} \stackrel{d}{\longrightarrow} P\left(\sum_{i=1}^{t} \gamma_{i}^{(c)}\right),
\end{array}
$$

где для $k=1, \ldots, t$

$$
\begin{aligned}
\gamma_{k} & =\left(c c_{2,1}\right)^{l_{2,1}^{(k)}}\left(2 c c_{2,2}\right)^{l_{2,2}^{(k)}} /\left(a_{k} q^{l^{(k)}}\right), \\
\gamma_{k}^{(c)} & =\left(c c_{2,1}\right)^{l_{2,1}^{(k)}}\left(2 c c_{2,2}\right)^{l_{2,2}^{(k)}} /\left(a_{k}^{(c)}\right) .
\end{aligned}
$$

Подсистемами в работе называются подсистемы реализаций случайной системы уравнений $S$. Несовместными 2-циклами будем называть 2-циклы реализаций случайного гиперграфа $M G(S)$, которые соответствуют несовместным двучленным подсистемам. Обозначим через $C_{l}^{(q)}=C_{l, n}^{(q)}$ и $C_{l}^{(0)}=C_{l, n}^{(0)}\left(C^{(q)}=C_{n}^{(q)}\right.$ и $\left.C^{(0)}=C_{n}^{(0)}\right)$ числа 2-циклов длины $l$ (всех длин) в случайном гиперграфе $M G(S)$, соответствующих подсистемам, которые имеют $q$ решений и несовместны, а через $C_{l}^{(c)}=C_{l, n}^{(c)}$ и $C^{(c)}=C_{n}^{(c)}$ - числа 2-циклов длины $l$ и всех длин в $M G\left(S^{(c)}\right)$, соответствующих подсистемам в реализациях случайной системы уравнений, имеющих $q$ решений относительно входящих в них переменных.

Теорема 4. Если $n \rightarrow \infty, M \sim c n, c>0-$ константа и $l_{1}, \ldots, l_{t}$ - различные натуральныле числа, $l_{k} \geqslant 2, k=1, \ldots, t$, то

$$
\begin{gathered}
\left(C_{l_{1}}^{(0)}, C_{l_{1}}^{(q)}, \ldots, C_{l_{t}}^{(0)}, C_{l_{t}}^{(q)}\right) \stackrel{d}{\longrightarrow} P\left(\mu_{l_{1}}^{(0)}, \mu_{l_{1}}^{(q)}, \ldots, \mu_{l_{t}}^{(0)}, \mu_{l_{t}}^{(q)}\right), \\
\left(C_{l_{1}}^{(c)}, \ldots, C_{l_{t}}^{(c)}\right) \stackrel{d}{\longrightarrow} P\left(\lambda_{l_{1}}, \ldots, \lambda_{l_{t}}\right),
\end{gathered}
$$

где для $k=1, \ldots, t$

$$
\begin{gathered}
\lambda_{l_{k}}=\left(\left(2 c c_{2}\right)^{l_{k}}+(-2 c \Delta)^{l_{k}}\right) /\left(4 l_{k}\right), \\
\mu_{l_{k}}^{(0)}=\lambda_{l_{k}}(1-1 / q), \quad \mu_{l_{k}}^{(q)}=\lambda_{l_{k}} / q,
\end{gathered}
$$

а при дополнительном условии $0<c<1 /\left(2 c_{2}\right)$

$$
\begin{gathered}
\left(C^{(0)}, C^{(q)}\right) \stackrel{d}{\longrightarrow} P\left(\mu^{(0)}, \mu^{(q)}\right), \\
C^{(c)} \stackrel{d}{\longrightarrow} P(\lambda) .
\end{gathered}
$$

Теорема 5. Если справедлива формула (3), то

$$
\begin{gathered}
\mathbf{P}\left(\zeta_{n}=0\right) \sim \mathbf{P}\left(C^{(0)}>0\right), \\
\mathbf{P}\left(\zeta_{n}=q^{C^{(q)}} \mid C^{(0)}=0\right) \sim 1, \quad \mathbf{P}\left(\zeta_{n}^{(c)}=q^{C^{(c)}}\right) \sim 1 .
\end{gathered}
$$

Обозначим через $\mathbf{P}_{c}(S)$ вероятность совместности случайной системы уравнений $S$, то есть сумму вероятностей ее совместных реализаций. 
Следствие 1. Если $n \rightarrow \infty, M \sim c n$, то $\mathbf{P}_{c}(S) \sim e^{-\mu^{(0)}}$ при условии (3); далее, $\underline{\lim }_{n \rightarrow \infty} \mathbf{P}_{c}(S) \leqslant e^{-\mu^{(0)}} n p u c^{*} \leqslant c<1 /\left(2 c_{2}\right) ; \mathbf{P}_{c}(S)=o(1) n p u c \geqslant 1 /\left(2 c_{2}\right)$.

Из теоремы 1 можно получить предельное распределение и моменты случайной величины $\zeta_{n}$ при условии совместности реализаций случайной системы уравнений $S$ : формулы для $\mathbf{P}\left(\zeta=q^{k}\right)$ и $\mathbf{E} \zeta^{r}$ надо разделить на $e^{-\mu^{(0)}}$ (предел вероятности совместности системы $S)$. Если $M=o(n)$ или $M \sim c n, 0<c<c^{*}, c_{2}=0$, то $\mathbf{P}\left(\zeta_{n}=1\right) \sim \mathbf{P}\left(\zeta_{n}^{(c)}=1\right) \sim 1$. При $q=2$ утверждения работы сохраняются, но $c_{2,1}=c_{2}, c_{2,2}=0, \Delta=-c_{2}$.

Совместность и среднее число решений различных видов случайных систем линейных уравнений исследовались в [2,4-11]. Г. В. Балакиным в конце 70-х годов в случае $c_{m}=1, m \geqslant 1$, оценено $\mathbf{E} \zeta_{n}^{(c)}$ и получены достаточные условия справедливости оценки $\mathbf{P}\left(\zeta_{n}>0\right) \sim 1$. При $q=2$ предельное распределение $\zeta_{n}^{(c)}$ и вероятность совместности системы $S$ с неравновероятной правой частью двучленных уравнений изучались в [11]; при $c_{2}=1$ сходимость распределений $\zeta_{n}$ и $\zeta_{n}^{(c)}$ доказана в [10], вероятность совместности системы $S$ с неравновероятной правой частью оценивалась в [9], с выборкой с возвращением - в [4, 7]. Книга В. Ф. Колчина [7] отличается от остальных работ систематическим использованием обобщенной схемы размещений и содержит две главы, посвященные случайным системам уравнений.

Применяемый в работе метод оценки характеристик случайных систем линейных уравнений позволяет распространить полученные результаты на системы уравнений с двучленными функциями, отличающимися от $y_{1}-y_{2}$ и $y_{1}+y_{2}$. При этом качественно результаты будут иметь такой же вид, но изменятся параметры $\mu^{(0)}, \mu^{(q)}, \lambda$, зависящие от вероятности того, что определитель матрицы $A_{l}$ в доказательстве теоремы 4 равен нулю.

\section{2. Доказательства}

Доказательство теоремь 3. Основная идея доказательства такая же, как и у доказательства теоремы 2 статьи [13] и теоремы 2.1 статьи [12], поэтому для облегчения восприятия структура доказательства и обозначения сохранены, насколько это оказалось возможным. В [13] рассматриваются гиперграфы $H_{j}$ более общего вида (строго сбалансированные), в [12] гиперграфы имеют метки ребер и являются гипердеревьями. В доказываемой теореме графы $H_{j}$ являются 2-циклами, которые строго сбалансированы, но их ребра имеют метки, как в [12], и в некоторых ребрах вершины упорядочены.

Через $\mathfrak{B}_{k}$ обозначим множество всех $H_{k}$-графов в реализациях рассматриваемого случайного гиперграфа, $k=1, \ldots, t$. Упорядочим элементы каждого из множеств $\mathfrak{B}_{k}$ некоторым образом и обозначим их $S\left(v_{i}^{(k)}\right), i=1, \ldots, b_{k}$, где $b_{k}=\left|\mathfrak{B}_{k}\right|$. Пусть $I\left(v_{i}^{(k)}\right)-$ случайная величина, равная индикатору события, заключающегося в том, что в случайном гиперграфе имеется $H_{k}$-граф $S\left(v_{i}^{(k)}\right)$. Тогда

$$
\xi_{k}=\sum_{i=1}^{b_{k}} I\left(v_{i}^{(k)}\right)
$$

и справедливо равенство (см. теорему 1.1 .4 в [7])

$$
\mathbf{E} \xi_{1}^{\left[r_{1}\right]} \ldots \xi_{t}^{\left[r_{t}\right]}=\sum \mathbf{E} I\left(v_{1}^{(1)}\right) \ldots I\left(v_{r_{1}}^{(1)}\right) \ldots I\left(v_{1}^{(t)}\right) \ldots I\left(v_{r_{t}}^{(t)}\right)
$$

где суммирование ведется по всем упорядоченным наборам $\bar{v}=\left(\bar{v}^{(1)}, \ldots, \bar{v}^{(t)}\right)$, для которых соответствующие им наборы подграфов принадлежат декартову произведению 
факториальных степеней множеств $\mathfrak{B}_{1}^{\left[r_{1}\right]} \times \ldots \times \mathfrak{B}_{t}^{\left[r_{t}\right]}$, а наборы $\bar{v}^{(k)}=\left(v_{1}^{(k)}, \ldots, v_{r_{k}}^{(k)}\right)$ соответствуют наборам подграфов, принадлежащих $\mathfrak{B}_{k}^{\left[r_{k}\right]}, k=1, \ldots, t$.

Разобьем эту сумму на суммы $\Sigma_{1}^{*}$ и $\Sigma_{2}^{*}$. К первой из них отнесем слагаемые, соответствующие наборам индексов $\bar{v}$, которым соответствуют гиперграфы $S\left(v_{1}^{(1)}\right), \ldots, S\left(v_{r_{1}}^{(1)}\right), \ldots$, $I\left(v_{1}^{(t)}\right), \ldots, I\left(v_{r_{t}}^{(t)}\right)$, множества вершин которых не пересекаются, а ко второй - все остальные наборы. Тогда

$$
\begin{aligned}
\Sigma_{1}^{*}=\left(\begin{array}{c}
n \\
r_{1} l^{(1)}
\end{array}\right) & \left(\begin{array}{c}
n-r_{1} l^{(1)} \\
r_{2} l^{(2)}
\end{array}\right) \cdots\left(\begin{array}{c}
n-r_{1} l^{(1)}-\ldots-r_{t-1} l^{(t-1)} \\
r_{t} l^{(t)}
\end{array}\right)\left(M_{2}\right)_{\sum_{k=1}^{t} r_{k} l^{(k)}} \\
& \times \prod_{k=1}^{t}\left(\frac{\left(r_{k} l^{(k)}\right) !}{\left(a^{(k)}\right)^{r_{k}}}\left(\left(\frac{c_{2,1}}{c_{2}}\right)^{l_{2,1}^{(k)}}\left(\frac{c_{2,2}}{c_{2}}\right)^{l_{2,2}^{(k)}} q^{-l^{(k)}}\left((n)_{2}\right)^{-l_{2,1}^{(k)}}\left(\begin{array}{l}
n \\
2
\end{array}\right)^{-l_{2,2}^{(k)}}\right)^{r_{k}}\right) .
\end{aligned}
$$

Здесь $\left(\begin{array}{c}n \\ r_{1} l^{(1)}\end{array}\right)\left(\begin{array}{c}n-r_{1} l^{(1)} \\ r_{2} l^{(2)}\end{array}\right) \ldots\left(\begin{array}{c}n-r_{1} l^{(1)}-\ldots-r_{t-1} l^{(t-1)} \\ r_{t} l^{(t)}\end{array}\right)$ - число способов выбора вершин для $r_{k}$ штук $H_{k}$-графов (2-циклов) без общих вершин; $\left(r_{k} l(k)\right) ! /\left(a^{(k)}\right)^{r_{k}}-$ числа различных способов размещения без пересечений $r_{k}$ упорядоченных $H_{k}$-графов на $r_{k} l^{(k)}$ вершинах, $k=1, \ldots, t$; величина $\left(M_{2}\right)_{\sum_{k=1}^{t} r_{k} l(k)}$ равна числу различных вариантов выбора упорядоченных множеств номеров для ребер, образующих рассматриваемые 2-циклы. Произведение этих величин дает число всех упорядоченных наборов $\bar{v}$, для которых соответствующие им наборы подграфов попарно не имеют общих вершин. Величина

$$
\left(\left(\frac{c_{2,1}}{c_{2}}\right)^{l_{2,1}^{(k)}}\left(\frac{c_{2,2}}{c_{2}}\right)^{l_{2,2}^{(k)}} q^{-l^{(k)}}\left((n)_{2}\right)^{-l_{2,1}^{(k)}}\left(\begin{array}{l}
n \\
2
\end{array}\right)^{-l_{2,2}^{(k)}}\right)^{r_{k}}
$$

равна вероятности того, что на соответствующих вершинах имеется $r_{k}$ штук $H_{k}$-графов, $k=1, \ldots, t$. Проведя необходимые преобразования, получаем, что

$$
\Sigma_{1}^{*} \sim \prod_{k=1}^{t}\left(\frac{n^{l^{(k)}}}{a^{(k)} q^{l^{(k)}}}\left(\frac{M c_{2,1}}{n^{2}}\right)^{l_{2,1}^{(k)}}\left(\frac{2 M c_{2,2}}{n^{2}}\right)^{l_{2,2}^{(k)}}\right)^{r_{k}} \sim \lambda_{1}^{r_{1}} \ldots \lambda_{t}^{r_{t}} .
$$

Можно убедиться, что $\Sigma_{2}^{*} \leqslant \Sigma_{2}$, где $\Sigma_{2}$ - аналогичная сумма в доказательстве теоремы 2 статьи [13] при рассмотрении 2-циклов, состоящих из ребер с неупорядоченными вершинами и не имеющих меток в случайном гиперграфе $M G_{n, \bar{M}}$. В [13] показано, что $\Sigma_{2}=o(1)$, поэтому $\Sigma_{2}^{*}=o(1)$. Следовательно, все смешанные факториальные моменты векторной случайной величины $\left(\xi_{1}, \ldots, \xi_{t}\right)$ сходятся к соответствующим факториальным моментам распределения $P\left(\lambda_{1}, \ldots, \lambda_{t}\right)$ и $\left(\xi_{1}, \ldots, \xi_{t}\right) \stackrel{d}{\longrightarrow} P\left(\gamma_{1}, \ldots, \gamma_{t}\right)$, так как многомерное распределение Пуассона однозначно определяется своими моментами (см., например, теорему 21 в гл. І книги [15]).

Сходимость распределения векторной случайной величины $\left(\xi_{1}^{(c)}, \ldots, \xi_{t}^{(c)}\right)$ доказывается аналогично. Единственное отличие - отсутствие в формулах для $\Sigma_{1}^{*}$ и $\Sigma_{2}^{*}$ величин $q^{l^{(j)}}$, так как значения правых частей уравнений однозначно определяются левыми частями уравнений. Сходимость распределений сумм случайных величин к соответствующим распределениям Пуассона следует из доказанной части теоремы и аддитивного свойства распределения Пуассона. 
Доказательство теоремы 4. Сначала докажем утверждение теоремы для случайного гиперграфа $M G(S)$. Подсистема реализации случайной системы линейных уравнений $S$ всегда имеет решения, если ей соответствует гиперграф без циклов, либо гиперграф с одним циклом, образованным ребрами, не все из которых состоят из двух вершин. Ниже будет доказано, что подсистема, соответствующая гиперграфу, имеющему ровно один 2-цикл, может иметь 0, 1 и $q$ решений. Сходимость распределения первой векторной случайной величины в доказываемом утверждении к многомерному распределению Пуассона с независимыми компонентами следует из теоремы 3. Получим оценки средних значений предельного распределения.

Расширенная матрица подсистемы из $l$ двучленных уравнений с помощью элементарных преобразований (умножение некоторых строк на $(-1)$ и перестановка столбцов) сводится к матрице $A_{l}^{*}$, которая с помощью эквивалентных преобразований сводится к матрице $A_{l, u_{l}}^{*}, u_{l}=0,2$, где

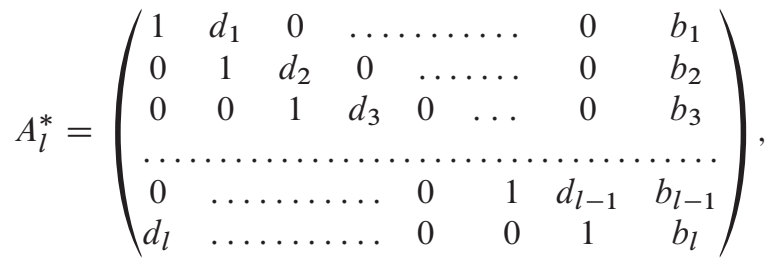

$$
\begin{aligned}
& A_{l, u_{l}}^{*}=\left(\begin{array}{ccccccc}
1 & 0 & \ldots \ldots & 0 & u_{1} & w_{1} \\
0 & 1 & 0 & \ldots & 0 & u_{2} & w_{2} \\
\ldots & \ldots & \ldots & \ldots & \ldots \ldots \ldots \ldots \ldots \\
0 & \ldots & 0 & 1 & 0 & u_{l-1} & w_{l-1} \\
0 & \ldots & \ldots & \ldots & 0 & u_{l} & w_{l}
\end{array}\right) \text {. }
\end{aligned}
$$

В каждой строке матрицы $A_{l}$, получающейся из матрицы $A_{l}^{*}$ в результате удаления последнего столбца, ровно по два ненулевых элемента: на главной диагонали стоят единицы, справа от них в первых $l-1$ строках - числа $d_{1}, \ldots, d_{l-1}$ соответственно, в последней строке первый элемент равен $d_{l}$. Здесь $d_{i}=1\left(d_{i}=-1=q-1\right)$, если в левой части соответствующего уравнения стоит функция $y_{1}+y_{2}\left(y_{1}-y_{2}\right)$. Для определителя матрицы $A_{l}$ справедливо равенство $\left|A_{l}\right|=1+(-1)^{l-1} d_{1} \ldots d_{l}=1-(-1)^{l+\delta}$, где $\delta=\delta\left(A_{l}\right)=0$, если среди чисел $d_{1}, \ldots, d_{l-1}$ четное число равно -1 и $\delta=1$ в остальных случаях. Следовательно, $\left|A_{l}\right|=2$, если $l+\delta$ нечетно и $\left|A_{l}\right|=0$, если $l+\delta$ четно.

Величина $w_{l}$ в матрице $A_{l, u_{l}}^{(p)}$ равна сумме значений правых частей уравнений рассматриваемой подсистемы $b_{1}, \ldots, b_{l}$ с коэффициентами 1 или $l$. В случайной системе уравнений $S$ правые части двучленных уравнений принимают значения $0,1, \ldots, q-1$ с равными вероятностями, поэтому на месте величины $w_{l}$ могут стоять значения $0,1, \ldots, q-1$ с равными вероятностями. Это позволяет описать распределение числа решений случайно выбираемой подсистемы двучленных уравнений в зависимости от четности числа уравнений $l$. Если $l$ четно, то при $\delta=1$ сумма $l+\delta$ нечетна, $\left|A_{l}\right|=u_{l}=2$ и число решений подсистемы равно 1 с вероятностью 1 при любых $w_{l}$; а при $\delta=0$ сумма $l+\delta$ четна, $\left|A_{l}\right|=u_{l}=0$ и число решений подсистемы равно 0 и $q$ при $w_{l} \neq 0$ и $w_{l}=0$ с вероятностями $1-1 / q$ и $1 / q$ соответственно. Если $l$ нечетно, то при $\delta=0$ сумма $l+\delta$ нечетна, $\left|A_{l}\right|=u_{l}=2$ и число решений подсистемы равно 1 с вероятностью 1 при любых $w_{l}$; а при $\delta=1$ сумма $l+\delta$ четна, $\left|A_{l}\right|=u_{l}=0$ и число решений подсистемы равно 0 и $q$ при $w_{l} \neq 0$ и $w_{l}=0$ с вероятностями $1-1 / q$ и $1 / q$ соответственно.

Получим оценки средних значений чисел несовместных 2-циклов при $M \sim c n, c>0$. 
Для четных $l$

$$
\mathbf{E} C_{l}^{(0)}=\left(\begin{array}{l}
n \\
l
\end{array}\right) \frac{l !}{2 l}\left(M_{2}\right)_{l} \frac{q-1}{q} \sum_{i=0}^{l / 2}\left(\left(\begin{array}{c}
l \\
2 i
\end{array}\right)\left(\frac{2 c_{2,1}}{c_{2}(n)_{2}}\right)^{2 i}\left(\frac{c_{2,2}}{c_{2}}\left(\begin{array}{l}
n \\
2
\end{array}\right)^{-1}\right)^{l-2 i}\right),
$$

где $\left(\begin{array}{l}n \\ l\end{array}\right)$ - число вариантов выбора $l$ неупорядоченных вершин для 2-цикла длины $l$; $l ! /(2 l)$ - число различных 2-циклов без меток с неупорядоченными вершинами в ребрах на выбранных $l$ вершинах; суммирование по четным $i$ обеспечивает перебор всех четных значений величины $\delta ;\left(\begin{array}{c}l \\ 2 i\end{array}\right)$ - число вариантов выбора четного числа $2 i$ ребер в циклах, соответствующих уравнениям с функцией $y_{1}-y_{2}, 2^{2 i}-$ число вариантов упорядочения вершин в этих ребрах; $\left(M_{2}\right)_{l}$ - число вариантов выбора номеров для $l$ ребер из $M_{2}$ возможных; $((q-1) / q)\left(c_{2,1} / c_{2}\right)^{2 i}\left(c_{2,2} / c_{2}\right)^{l-2 i}\left((n)_{2}\right)^{-2 i}\left(\begin{array}{c}n \\ 2\end{array}\right)^{-(l-2 i)}-$ вероятность того, что на соответствующих вершинах имеется несовместный 2-цикл. Следовательно,

$$
\mathbf{E} C_{l}^{(0)} \sim \frac{(q-1)(2 c)^{l}}{2 l q} \sum_{i=0}^{l / 2}\left(\left(\begin{array}{c}
l \\
2 i
\end{array}\right)\left(c_{2,1}\right)^{2 i}\left(c_{2,2}\right)^{l-2 i}\right) \sim \frac{q-1}{4 l q}\left(\left(2 c c_{2}\right)^{l}+(-2 c \Delta)^{l}\right) .
$$

Такая же оценка для нечетных $l$ получается из формулы

$$
\mathbf{E} C_{l}^{(0)}=\frac{(n)_{l}(q-1)}{2 q l}\left(M_{2}\right)_{l} \sum_{i=0}^{(l-1) / 2}\left(\left(\begin{array}{c}
l \\
2 i+1
\end{array}\right)\left(\frac{c_{2,1}}{c_{2}(n)_{2}}\right)^{2 i+1}\left(\frac{c_{2,2}}{c_{2}}\left(\begin{array}{l}
n \\
2
\end{array}\right)^{-1}\right)^{l-2 i-1}\right) .
$$

Предельные значения для средних значений чисел 2-циклов, соответствующих подсистемам с $q$ решениями, получаются из приведенных выше формул в результате замены множителя $1-1 / q$ на $1 / q$.

Сходимость распределения векторной случайной величины $\left(C_{l_{1}}^{(0)}, C_{l_{1}}^{(q)}, \ldots, C_{l_{s}}^{(0)}, C_{l_{s}}^{(q)}\right)$ доказана. Отсюда и из леммы 2.4.3 в [7] следует сходимость распределения случайной величины $\left(C^{(0)}, C^{(q)}\right)$, оценки для средних значений компонент предельного распределения которой удовлетворяют равенствам

$$
\mu^{(0)}=\frac{q-1}{4 q l} \sum_{l=2}^{\infty}\left(\left(2 c c_{2}\right)^{l}+(-2 c \Delta)^{l}\right), \quad \mu^{(q)}=\mu^{(0)} /(q-1) .
$$

Свернув ряды, получаем оценки величин $\mu^{(q)}$ и $\mu^{(0)}$, приведенные перед теоремой 1 .

Сходимость распределения векторной случайной величины $\left(C_{l_{1}}^{(c)}, \ldots, C_{l_{t}}^{(c)}\right)$ к многомерному распределению Пуассона следует из теоремы 3. В этом случае расширенная матрица двучленной подсистемы с помощью элементарных преобразований приводится к виду $A_{l}^{*}$, и формула для $\left|A_{l}\right|$ сохраняется. Матрица $A_{l}^{*}$ эквивалентна $A_{l, u_{l}}^{*}, u_{l}=0,2$. Двучленная подсистема совместна, поэтому при $u_{l}=0$ она имеет $q$ решений, так как в этом случае $w_{l}=0$; при $u_{l}=2$ она всегда имеет одно решение. Поэтому распределение числа решений случайно выбираемой подсистемы двучленных уравнений в зависимости от четности числа уравнений $l$ описывается следующим образом. Если $l$ четно, то при $\delta=1$ сумма $l+\delta$ нечетна, $\left|A_{l}\right|=u_{l}=2$ и подсистема всегда имеет одно решение; а при $\delta=0$ сумма $l+\delta$ четна, $\left|A_{l}\right|=u_{l}=0$ и подсистема всегда имеет $q$ решений. Если $l$ нечетно, то при $\delta=0$ сумма $l+\delta$ нечетна, $\left|A_{l}\right|=u_{l}=2$ и подсистема всегда имеет 
одно решение; а при $\delta=1$ сумма $l+\delta$ четна, $\left|A_{l}\right|=u_{l}=0$ и подсистема всегда имеет $q$ решений.

Оценим $\mathbf{E} C_{l}^{(c)}$ при $M \sim c n, c>0$. Для четных $l$

$$
\mathbf{E} C_{l}^{(c)}=\frac{(n)_{l}}{2 l}\left(M_{2}\right)_{l} \sum_{i=0}^{l / 2}\left(\left(\begin{array}{c}
l \\
2 i
\end{array}\right)\left(\frac{2 c_{2,1}}{c_{2}(n)_{2}}\right)^{2 i}\left(\frac{2 c_{2,2}}{C_{2}(n)_{2}}\right)^{l-2 i}\right) \sim \frac{\left(2 c c_{2}\right)^{l}+(-2 c \Delta)^{l}}{4 l} .
$$

При нечетных $l$ справедлива такая же оценка, получаемая из формулы

$$
\mathbf{E} C_{l}^{(c)}=\frac{(n)_{l}}{2 l}\left(M_{2}\right)_{l} \sum_{i=0}^{(l-1) / 2}\left(\left(\begin{array}{c}
l \\
2 i+1
\end{array}\right)\left(\frac{2 c_{2,1}}{c_{2}(n)_{2}}\right)^{2 i+1}\left(\frac{2 c_{2,2}}{c_{2}(n)_{2}}\right)^{l-2 i-1}\right) .
$$

Сходимость распределения векторной случайной величины $\left(C_{l_{1}}^{(c)}, \ldots, C_{l_{t}}^{(c)}\right)$ доказана. Из этого следует, что $C_{2}^{(c)}+\ldots+C_{t}^{(c)} \stackrel{d}{\longrightarrow} P\left(\lambda_{2}+\ldots+\lambda_{t}\right), t=2,3, \ldots$ Отсюда и из леммы 2.4.3 в книге [7] следует сходимость распределения случайной величины $C^{(c)}$, среднее значение предельного распределения удовлетворяет формуле

$$
\lambda=\lim _{n \rightarrow \infty} C^{(c)}=\sum_{l=2}^{\infty} \frac{\left(2 c c_{2}\right)^{l}+(-2 c \Delta)^{l}}{4 l}=-\frac{1}{4} \log \left(\left(1-2 c c_{2}\right)(1+2 c \Delta)\right)-c c_{2,1} .
$$

Доказательство теоремы 4 закончено.

Доказательство теоремы 5. При условии (3) со стремящейся к единице вероятностью реализации случайных систем уравнений $S$ и $S^{(c)}$ соответствуют гиперграфам, состоящим только из гипердеревьев и компонент с одним циклом. Уравнения каждой такой реализации можно переупорядочить так, что каждое уравнение имеет с предыдущими уравнениями не более одной общей переменной, кроме случаев, когда соответствующее ему ребро сопоставленного гиперграфа замыкает цикл. Переупорядочение уравнений не влияет на число решений реализации. Число решений переупорядоченных вышеприведенным способом реализаций случайных систем уравнений, состоящих только из гипердеревьев и компонент с одним циклом, сокращается в $q$ раз при добавлении каждого уравнения, кроме случаев образования 2-циклов в сопоставленном системе уравнений гиперграфе. Далее доказательства для случайных систем уравнений $S$ и $S^{(c)}$ различаются.

При появлении в реализации $M G(S)$ несовместного 2-цикла соответствующая ей реализация $S$ становится несовместной. Отсюда, $\mathbf{P}\left(\zeta_{n}=0\right) \sim \mathbf{P}\left(C^{(0)}>0\right)$. При образовании 2-цикла, соответствующего совместной подсистеме, число решений может изменяться различным образом. В соответствии с построенной в доказательстве теоремы 3 классификацией числа решений системы двучленных уравнений, такие 2-циклы могут соответствовать только подсистемам с $q$ или с одним решением. При добавлении ребра, замыкающего 2-цикл, число решений соответствующей реализации случайной системы в первом случае не изменяется, во втором сокращается в $q$ раз. Вероятность того, что реализации случайной системы уравнений $S$ соответствуют реализациям случайного гиперграфа $M G(S)$, состоящим только из гипердеревьев и компонент с одним циклом, стремится к единице при $n \rightarrow \infty$. При условии $C^{(0)}=0$ такие реализации имеют в $q^{M-C^{(q)}}$ раз меньше решений, чем реализация из $M=0$ уравнений, имеющая $q^{n}$ решений. Отсюда следует, что $\mathbf{P}\left(\zeta_{n}=q^{C^{(q)}} \mid C^{(0)}=0\right) \sim 1$.

Если при добавлении уравнения в реализацию случайной системы $S^{(c)}$ в соответствующем ей гиперграфе образуется 2-цикл, то число решений такой реализации либо 
сокращается в $q$ раз (если этот 2-цикл соответствует двучленной подсистеме с одним решением), либо не изменяется (если этот 2-цикл соответствует двучленной подсистеме с $q$ решениями). Следовательно, со стремящейся к единице при $n \rightarrow \infty$ вероятностью, реализации случайной системы $S^{(c)}$ имеют в $q^{M-C^{(c)}}$ раз меньше решений, чем реализация из $M=0$ уравнений, имеющая $q^{n}$ решений. Отсюда следует, что $\mathbf{P}\left(\zeta_{n}^{(c)}=q^{C^{(c)}}\right) \sim 1$. Теорема 5 доказана.

Доказательство теорем 1, 2. Утверждение о сходимости по распределению $\zeta_{n}$ к $\zeta$ и $\zeta_{n}^{(c)}$ к $\zeta^{(c)}$, вид распределений и моментов случайных величин $\zeta, \zeta^{(c)}$ следуют из теорем 4 и 5 . Так как $\zeta_{n} \geqslant 0$, для любого фиксированного $t=1,2, \ldots$

$$
\begin{aligned}
\varliminf_{n \rightarrow \infty} \mathbf{E} \zeta_{n}^{r} & =\underline{\lim }_{n \rightarrow \infty} \sum_{k=0}^{n} \mathbf{P}\left(\zeta_{n}=q^{r}\right) q^{r k} \geqslant \lim _{n \rightarrow \infty} \sum_{k=0}^{t} \mathbf{P}\left(\zeta_{n}=q^{r}\right) q^{r k} \\
& =\sum_{k=0}^{t} \mathbf{P}\left(\zeta=q^{r}\right) q^{r k}=e^{-\mu^{(0)}-\mu^{(q)}} \sum_{k=0}^{t} \frac{\left(\mu^{(q)} q^{r}\right)^{k}}{k !} .
\end{aligned}
$$

Ряд $\sum_{k=0}^{\infty}\left(\mu^{(q)} q^{r}\right)^{k} / k$ ! в рассматриваемых условиях сходится и равен $e^{\mu^{(q)} q^{r}}$, поэтому для любого наперед заданного сколь угодно малого положительного числа $\varepsilon$ можно выбрать такое $t$, что

$$
e^{-\mu^{(0)}+\mu^{(q)}\left(q^{r}-1\right)}-e^{-\mu^{(0)}-\mu^{(q)}} \sum_{k=0}^{t} \frac{\left(\mu^{(q)} q^{r}\right)^{k}}{k !}<\varepsilon .
$$

Следовательно, справедлива нижняя оценка

$$
\varliminf_{n \rightarrow \infty} \mathbf{E} \zeta_{n}^{r} \geqslant e^{-\mu^{(0)}+\mu^{(q)}\left(q^{r}-1\right)}=\mathbf{E} \zeta^{r}
$$

Если условие В выполняется для всех случайных уравнений случайной системы $S$, то $\mathbf{E} \zeta_{n}=1$, а из формулы для $\mathbf{E} \zeta^{r}$ следует, что $\mathbf{E} \zeta=1$. Из сходимости $\mathbf{E} \zeta_{n}$ к $\mathbf{E} \zeta$ следует сходимость и всех остальных моментов (см. стр. 52 в [3]).

Для доказательства сходимости моментов случайной величины $\mathbf{E} \zeta_{n}^{(c)}$ введем вспомогательную случайную заведомо совместную систему уравнений $S^{(c, *)}$, соответствующую смыслу определения 4.1 в [1]. Система $S^{(c, *)}$ состоит из $q^{n}$ случайных заведомо совместных систем уравнений $S^{(c)}=S^{(c)}(\mathbf{b})$, в которых истинное решение $\mathbf{b}$ пробегает все $q^{n}$ возможных значений. Распределения чисел решений $\zeta_{n}^{(c)}$ и $\zeta_{n}^{(c, *)}$ систем $S^{(c)}$ и $S^{(c, *)}$ одинаковы, так как распределение числа решений $S^{(c)}(\mathbf{b})$ инвариантно относительно значения истинного решения $\mathbf{b}$. Определенная таким образом система $S^{(c, *)}$ согласована со случайной системой уравнений $S$, удовлетворяющей условию В. Понятие согласованных систем уравнений введено в определении 4.1 в [1], выполнение условий 1, 2 которого следует из леммы 4.1 этой же работы и условия $\mathbf{B}$, а справедливость условия 3, заключающегося в инвариантности распределения случайной величины $\zeta_{n}^{(c)}$ относительно истинного решения $\mathbf{b}$ случайной системы уравнений $S^{(c)}(\mathbf{b})$, отмечена выше. Формула (4.9) в [1] дает основное соотношение для моментов чисел решений согласованных систем уравнений

$$
\mathbf{E} \zeta_{n}^{r}=\mathbf{E} \zeta_{n} \mathbf{E}\left(\zeta_{n}^{(c, *)}\right)^{r-1}, \quad r=2,3, \ldots
$$


откуда

$$
\mathbf{E}\left(\zeta_{n}^{(c, *)}\right)^{r}=\mathbf{E} \zeta_{n}^{r+1} / \mathbf{E} \zeta_{n}, \quad r=1,2, \ldots
$$

Воспользовавшись полученными в теореме 1 оценками моментов случайной величины $\zeta_{n}$ и отмеченным выше совпадением распределений случайных величин $\zeta_{n}^{(c)}$ и $\zeta_{n}^{(c, *)}$, убеждаемся, что $\mathbf{E}\left(\zeta_{n}^{(c)}\right)^{r} \sim e^{q \mu^{(q)}\left(q^{r}-1\right)}, r=1,2, \ldots$, так как $\lambda=q \mu^{(q)}$. Поэтому в рассматриваемых условиях $\mathbf{E}\left(\zeta_{n}^{(c)}\right)^{r} \rightarrow \mathbf{E}\left(\zeta^{(c)}\right)^{r}, r=1,2, \ldots$

Следствие 1 вытекает из сходимости $C^{(0)} \stackrel{d}{\longrightarrow} P\left(\mu^{(0)}\right)$, доказанной в теореме 4, и геометрических свойств системы $S$.

\section{Список литературы}

1. Балакин Г. В., Введение в теорию случайных систем уравнений. В сб.: Труды по дискретной математике, 1, 1997, с. 1-18.

2. Балакин Г. В., Колчин В. Ф., Хохлов В. И., Гиперциклы в случайном гиперграфе. Дискретная математика (1991) 3, №3, 102-108.

3. Биллингсли П., Сходимость вероятностных мер. Наука, Москва, 1977.

4. Колчин В. Ф., Системы случайных уравнений. МИЭМ, Москва, 1988.

5. Колчин В. Ф., О пороговом эффекте для систем случайных уравнений. Труды по дискретной математике (1998) 2, 183-190.

6. Колчин В. Ф., Пороговое свойство для систем уравнений в конечных полях. Дискретная математика (1999) 11, №3, 15-23.

7. Колчин В. Ф., Случайные графы. Физматлит, Москва, 2004.

8. Колчин В. Ф., Хохлов В. И., Пороговый эффект для систем случайных уравнений специального вида. Дискретная математика (1995) 7, №4, 29-39.

9. Шаповалов А. В., Вероятность совместности случайных систем булевых уравнений. Дискретная математика (1995) 7, №3, 146-159.

10. Shapovalov A. V., The number of decisions of random monomial and binomial linear systems of equations. In: Probabilistic Methods in Discrete Mathematics. VSP, Utrecht, 1997, pp. 333-342.

11. Shapovalov A. V., Characteristics of random systems of Boolean equations with non-regular left-hand side. In: Probabilistic Methods in Discrete Mathematics. VSP, Utrecht, 2002, pp. 345-350.

12. Шаповалов А. В., Пороговые функции совместности случайных систем уравнений. Труды по дискретной математике (2006) 9, 377-400.

13. Шаповалов А. В., Распределения чисел конечных подграфов в случайных неоднородных гиперграфах. Дискретная математика (2006) 18, №3, 102-114.

14. Шаповалов А. В., Цикловая структура случайного неоднородного гиперграфа на докритическом этапе эволюции. Дискретная математика (2007) 19, №4, 52-69.

15. Bollobás B., Random graphs. Academic Press, London, 1985.

Статья поступила 13.08.2008. Переработанный вариант поступил 2.10.2008. 